\title{
CONTRIBUTION TO THE KNOWLEDGE OF THE BUTTERFLY AND SKIPPER FAUNA OF NORTHERN DALMATIA MAINLAND
}

\author{
Rudi Verovnik ${ }^{1}$, Toni Koren ${ }^{2}$ \& Gordana Glavan ${ }^{1}$ \\ ${ }^{1}$ University of Ljubljana, Biotechnical Faculty, Department of Biology, Jamnikarjeva 101, \\ 1000 Ljubljana, Slovenia \\ ${ }^{2}$ Croatian Herpetological Society - Hyla, I. Lipovac 7, 10000 Zagreb, Croatia
}

Verovnik, R., Koren, T. \& Glavan, G.: Contribution to the knowledge of the butterfly and skipper fauna of northern Dalmatia mainland. Nat. Croat., Vol. 24, No. 2., 265-280, Zagreb, 2015.

The mainland of northern Dalmatia has been surprisingly omitted from recent faunistic studies of butterfly fauna in the Croatian coastal region. In order to fill this gap we summarized our observations made in the regionduring the last two decades. Altogether 81 species were recorded, including several rare and potentially threatened species. Among these the distribution of the following species is discussed in detail: Carcharodus orientalis, Gegenes pumilio, Euchloe ausonia, Cacyreus marshalli, Tarucus balkanica, Polyommatus thersites, Euphydryas aurinia, Hyponephele lupina, and Proterebia afra. Although the butterfly diversity and conservation value of the region are not exceptionally high, some locations, in particular valleys of the Bijelina and Karišnica, could be designated as micro-reserves for conservation of high butterfly diversity including the majority of the threatened species in the region. Abandonment and subsequent overgrowing together with habitat destruction due to building of infrastructure and extensions of urban areas have been recognised as the most important factors causing butterfly declines in the region.

Key words Lepidoptera, Papilionoidea \& Hesperioidea, distribution, diversity, conservation

Verovnik, R., Koren, T. \& Glavan, G.: Prilog poznavanju faune leptira kopnenog dijela sjeverne Dalmacije. Nat. Croat., Vol. 24, No. 2., 265-280, Zagreb, 2015.

Kopneni dio sjeverne Dalmacije iznenađujuće je izostavljen iz recentnih faunističkih istraživanja leptira obalnog dijela Hrvatske. Kako bi upotpunili tu prazninu, objedinili smo podatke i opažanja iz ovog područja prikupljene u zadnja dva desetljeća. Sveukupno smo zabilježili 81 vrstu leptira, uključujući i nekoliko rijetkih i potencijalno ugroženih vrsta. U radu detaljnije raspravljamo o rasprostranjenosti sljedećih vrsta: Carcharodus orientalis, Gegenes pumilio, Euchloe ausonia, Cacyreus marshalli, Tarucus balkanicus, Polyommatus thersites, Euphydryas aurinia, Hyponephele lupina i Proterebia afra. Iako zabilježena raznolikost leptira i očuvanost regije nije izrazito visoka, neka područja, posebice doline Bijeline i Karišnice mogu biti označene kao područja mikro-rezervata za očuvanja visoke raznolikosti leptira, uključujući i većinu ugroženih vrsta iz regije. Napuštanje i naknadno zarastanje zajedno s uništavanjem staništa zbog izgradnje infrastrukture i proširenja urbanih područja prepoznati su kao najvažniji čimbenici koji uzrokuju opadanje brojnosti leptira u regiji.

Ključne riječi Lepidoptera, Papilioniodea \& Hesperioidea, rasprostranjenost, raznolikost, očuvanje

\section{INTRODUCTION}

Dalmatia was among first regions in Croatia to be studied, with published butterfly records, as early as in the $19^{\text {th }}$ century (GERMAR, 1814; MANN, 1864). The beginning of the $20^{\text {th }}$ century brought even more entomological activities with detailed surveys published by Abafi - Aigner \& Pável (1900), Buresch \& Iltschew (1915), Stauder (1911, 1913, 1921, 
1922, 1923), Schwingenschuss \& Wagner (1925), and Galvagni (1902, 1909). During the last few decades a multitude of faunistic papers were published covering both the coastal and mainland parts of Croatia. The Croatian islands in particular were the focus of many studies (Withrington \& Verovnik, 2008; Withrington, 2008; Koren, \& Bjelić, 2010; Kučinić et al., 2010; Koren et al., 2010; VerovniK, 2011; Koren et al., 2015a). However, surprisingly little was published on the fauna of butterflies of northern Dalmatia, especially for the coastal part. Records from few sites from the region were published by Habeler (1976) and Wiemers (1982). Additional records come from the catalogue of the collection of former Croatian national zoological museum in Zagreb (MLAdinov, 1973, 1975). A more comprehensive study was published on the fauna of the Zrmanja River (Koren et al., 2011), a neighbouring region to the surveyed area.

In order to fill the evident gap in the published records of butterflies in northern Dalmatia we have summarized almost two decades of observations and provide a discussior of the more interesting species, especially of those whose distribution reaches its northern limit in the studied region.

\section{MATERIAL AND METHODS}

Northern Dalmatia stretches from the Silba, Olib, and Pag Islands in the northwest and as far south as Primošten (Rogoznica Peninsula) in the southeast (Magaš, 2013). The coastal part is wide compared to other parts of Dalmatia with plains named 'Ravni kotari' covering most of the region. These plains are mainly composed of flysch sedimentary rocks with brown soil suitable for agriculture. Thy regions bordering o Ravni kotari, as well as the neighbouring islands, are built of limestone. The coastal region of

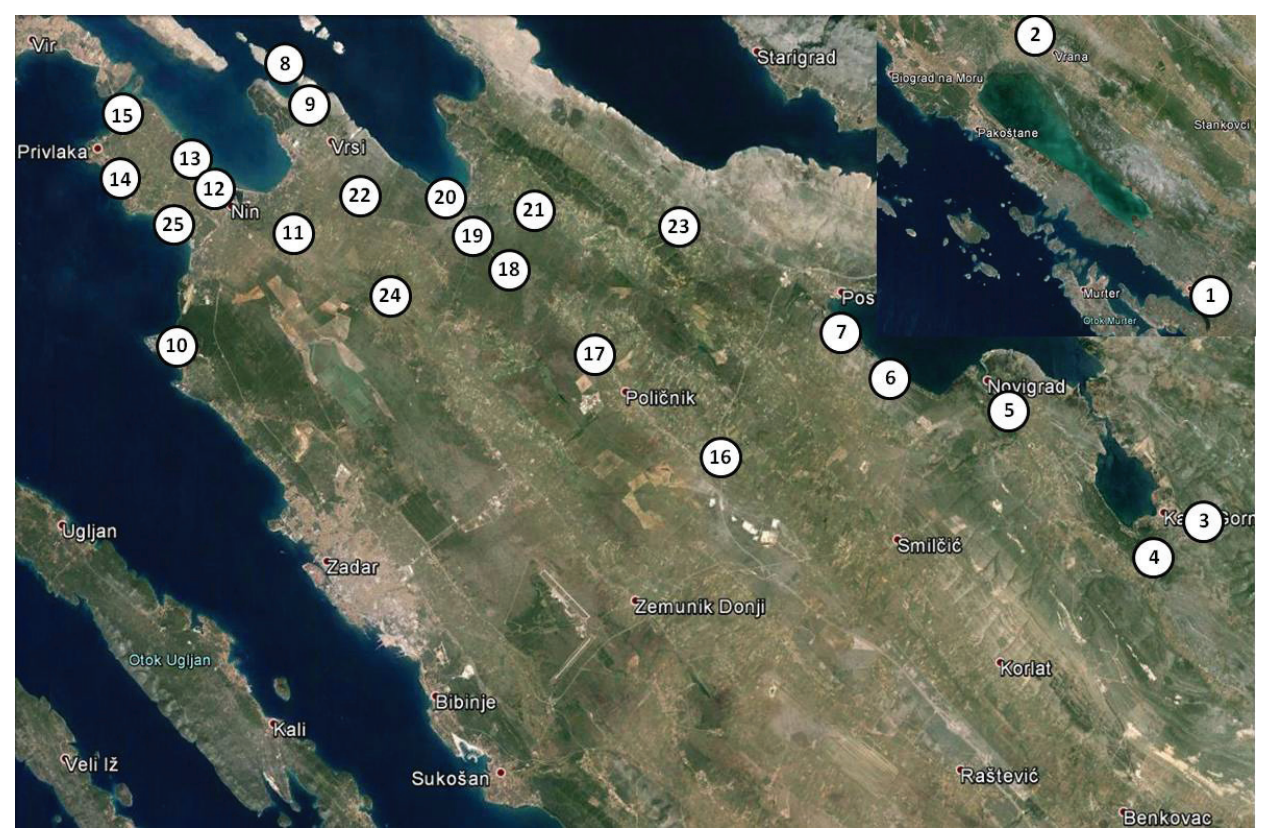

Fig. 1. Map of sampling locations where butterflies were surveyed in northern Dalmatia. The right corner map shows dislocated part of the survey area at Vrana lake. 
Northern Dalmatia has a typical Mediterranean climate with hot dry summers. The former Eumediterranean evergreen vegetation of Holm oak and Aleppo pine woods has been long degraded into secondary shrubs like garrigue or macchia, however most of northern Dalmatia is under cultivation with orchards and pastures (MAGAš, 2013).

Altogether, 25 sites were visited in northern Dalmatia (see Fig. 1 and the list below), those with butterfly-rich habitats, most of the localities on several occasions and in different seasons (see Tab. 1). Prior to field surveys, Google Earth aerial photos were examined and potential sites were selected. Butterflies were netted for identification and then released. Butterflies of the genus Leptidea were not identified to species level, and were recorded as Leptidea sinapis/juvernica, although the presence of L. sinapis (Linnaeus, 1758) is much more plausible in Dalmatia given the known distribution of L. juvernica (Williams, 1946) (Dinca et al., 2011). The genitalia of Hipparchia spp. were checked according to LAFRANCHIs (2004), without harming the butterflies. Determination of Melitaea phoebe ([Denis \& Schiffermüller], 1775) and M. ornata Christoph, 1893 was based on external characteristics only (wing patterns, shape of the distal part of the antennae) (Тóтн \& VARGA, 2011). Nomenclature follows the Fauna Europaea list of European butterflies (Fauna Europaea, 2015). The surveyed localities are:

1. Pirovac, at a spring near the main road just $S$ of the town; $43^{\circ} 49^{\prime} 1.35^{\prime \prime} \mathrm{N}, 15^{\circ} 41^{\prime} 7.39^{\prime \prime} \mathrm{E}$; maquis, flowery road verges.

2. Vrana, below rocky slopes at a strong spring E of the village; $43^{\circ} 57^{\prime} 32.92^{\prime \prime} \mathrm{N}$, $15^{\circ} 33^{\prime} 11.73^{\prime \prime} \mathrm{E}$; abandoned grasslands, wet sands along the road, rocky and bushy slopes.

3. Karin, Bijelina Valley, middle part; $44^{\circ} 7^{\prime} 57.33^{\prime \prime} \mathrm{N}, 15^{\circ} 38^{\prime} 54.38^{\prime \prime} \mathrm{E}$; dry stream bed, bushes, dry rocky grasslands.

4. Karin, Karišnica Valley, middle part; $44^{\circ} 7^{\prime} 13.15^{\prime \prime} \mathrm{N}, 15^{\circ} 37^{\prime} 43.30^{\prime \prime} \mathrm{E}$; dry river bed, abandoned dry grasslands, path through mixed woods.

5. Novigrad, Pridraga, side road at the entrance to the gorge NE of the village; $44^{\circ} 10^{\prime} 14.14^{\prime \prime} \mathrm{N}, 15^{\circ} 33^{\prime} 29.18^{\prime \prime} \mathrm{E}$; hay meadows, hedges, orchards

6. Novigrad, along the road to Posedarje near the turn for Podgradina; $44^{\circ} 11^{\prime} 15.20^{\prime \prime} \mathrm{N}$, $15^{\circ} 29^{\prime} 39.04^{\prime \prime} \mathrm{E}$; small rocky gully, partially overgrown grasslands

7. Posedarje, slope above the road to Novigrad S of the town; $44^{\circ} 11.977^{\prime} \mathrm{N}, 15^{\circ} 28.746^{\prime} \mathrm{E}$, dry rocky grasslands partially overgrown with juniper bushes, small gully with overgrown grassland.

8. Vrsi, Jasenovo, on the Grudina Peninsula; $44^{\circ} 17^{\prime} 05.30^{\prime \prime} \mathrm{N}, 15^{\circ} 13^{\prime} 14.70^{\prime \prime} \mathrm{E}$; rocky pastures.

9. Vrsi, Jasenovo, surroundings of a small church; $44^{\circ} 16^{\prime} 45.71^{\prime \prime} \mathrm{N}, 15^{\circ} 13^{\prime} 16.52^{\prime \prime} \mathrm{E}$; dry rocky pastures, hay meadows, abandoned fields.

10. Petrčani, above Punta Skala resort; $44^{\circ} 11^{\prime} 32.65^{\prime \prime} \mathrm{N}, 15^{\circ} 9^{\prime} 9.01^{\prime \prime} \mathrm{E}$; maquis, small grassy and rocky patches along paths.

11. Nin, slopes $S$ of the small valley of the Miljašića River; $44^{\circ} 14^{\prime} 2.80^{\prime \prime} \mathrm{N}, 15^{\circ} 12^{\prime} 38.91^{\prime \prime} \mathrm{E}$; dry rocky grasslands with small gullies, humid hay meadows in the valley.

12. Nin, Ninska laguna, SW edge of the lagoon; $44^{\circ} 14^{\prime} 50.35^{\prime \prime} \mathrm{N}, 15^{\circ} 10^{\prime} 32.50^{\prime \prime} \mathrm{E}$; salty marshes, dry grasslands.

13. Privlaka, Sabunike, sandy beach southeast of the village; $44^{\circ} 15^{\prime} 13.82^{\prime \prime} \mathrm{N}$, $15^{\circ} 10^{\prime} 8.76^{\prime \prime} \mathrm{E}$; partially overgrown sandy areas, bushes.

14. Privlaka, small peninsula $S$ of Batalaža; $44^{\circ} 15^{\prime} 5.42^{\prime \prime} \mathrm{N}, 15^{\circ} 7^{\prime} 34.12^{\prime \prime} \mathrm{E}$, dry rocky grasslands, pine woods, orchards.

15. Privlaka, on the mainland $S$ of the bridge to Vir; $44^{\circ} 16^{\prime} 21.08^{\prime \prime} \mathrm{N}, 15^{\circ} 7^{\prime} 53.62^{\prime \prime} \mathrm{E}$; humid grasslands. 
16. Suhovare, E from the village, near the stream and Vlačine lake; $44^{\circ} 09^{\prime} 23.5^{\prime \prime} \mathrm{N}$ $15^{\circ} 25^{\prime} 22.1^{\prime \prime} \mathrm{E}$; dry grasslands, forest edge.

17. Poličnik, grasslands around the village; $44^{\circ} 11^{\prime} 10.2^{\prime \prime} \mathrm{N} 15^{\circ} 22^{\prime} 00.5^{\prime \prime} \mathrm{E}$; dry karstic pastures.

18. Poljice, grasslands $2 \mathrm{~km}$ E from the village; $44^{\circ} 13^{\prime} 15.6^{\prime \prime} \mathrm{N} 15^{\circ} 18^{\prime} 50.7^{\prime \prime} \mathrm{E}$; dry karstic pastures and grasslands.

19. Gajine, grasslands $\mathrm{N}$ of village Poljice; $44^{\circ} 13^{\prime} 53.1^{\prime \prime} \mathrm{N} 15^{\circ} 18^{\prime} 08.5^{\prime \prime} \mathrm{E}$; dry rocky grasslands.

20. Ljubač Stanovi, near the Krnjeza River; $44^{\circ} 14^{\prime} 37.1^{\prime \prime} \mathrm{N} 15^{\circ} 17^{\prime} 33.7^{\prime \prime} \mathrm{E}$; salty marshes, dry grasslands.

21. Čolaci, pastures E from the Krnjeza River; $44^{\circ} 14^{\prime} 16.9^{\prime \prime} \mathrm{N} 15^{\circ} 20^{\prime} 10.5^{\prime \prime} \mathrm{E}$; dry karstic grasslands.

22. Dumerača, E from Ljubač-Stanovi village; $44^{\circ} 14^{\prime} 20.5^{\prime \prime} \mathrm{N} 15^{\circ} 15^{\prime} 11.4^{\prime \prime} \mathrm{E}$; dry rocky grasslands.

23. Jovići, grasslands and pastures $S$ from the village; $44^{\circ} 13^{\prime} 52.2^{\prime \prime} \mathrm{N} 15^{\circ} 24^{\prime} 25.9^{\prime \prime} \mathrm{E}$; dry rocky grasslands.

24. Žerava, grasslands near the village; $44^{\circ} 12^{\prime} 23.2^{\prime \prime} \mathrm{N} 15^{\circ} 15^{\prime} 22.9^{\prime \prime} \mathrm{E}$; dry karstic pastures and bushy areas.

25. Privlaka, Škrapavac, grasslands at cape Kremenjača; $44^{\circ} 13.940^{\prime} \mathrm{N}, 15^{\circ} 9.331^{\prime} \mathrm{E}$; extensive flower rich grasslands and sandy paths

\section{RESULTS}

During our surveys in northern Dalmatia spanning almost two decades, 81 butterfly species were recorded, representing approximately $40 \%$ of the butterfly fauna of Croatia (ŚAšić \& Minoci, 2011; Koren \& ŠTiH, 2013; Koren et al., 2013a, 2013b). The systematic list along with the presence on each locality is shown in Tab 1.

Tab. 1. List of recorded butterfly species from northern Dalmatia. Localities are numbered as in the Methods section. Observation dates are given in brackets following each locality. If a species was seen more than three times at one locality only the first and the last date of the observation are given.

\begin{tabular}{|c|c|}
\hline Species & Locality and date of observation \\
\hline \multicolumn{2}{|l|}{ HESPERIIDAE } \\
\hline Erynnis tages (Linnaeus, 1758) & $\begin{array}{l}4 \text { (3.V.2008), } 9 \text { (27.IV.2008), } 11 \text { (28.IV.2008, 17.VIII.2008), } 13 \\
\text { (1.V.2007), } 14 \text { (12.VIII.2007), } 15 \text { (30.IV.2008), } 21 \text { (7.V.2015), } 22 \\
\text { (7.V.2015) }\end{array}$ \\
\hline Carcharodus alceae (Esper, [1780]) & $\begin{array}{l}1 \text { (13.IX.2002), } 2 \text { (10.IX.2004, 5.VI.2010), } 3 \text { (26.VIII.2009- 19.IX.2015), } \\
4 \text { (19.VIII.2007, 24.VIII.2012), 7 (3.V.2013, 21.VIII.2014), 9 (27.IV.2008), } \\
10 \text { (13.VIII.2001, 15.VIII.2007, 10.VIII.2011), } 11 \text { (28.IV.2008, } \\
\text { 17.VIII.2008), 14 (11.VIII.2005-15.VIII.2015), } 18 \text { (7.V.2015) }\end{array}$ \\
\hline $\begin{array}{l}\text { Carcharodus floccifera (Zeller, } \\
\text { 1847) }\end{array}$ & 3 (6.VI.2010), 11 (6.VI.2010) \\
\hline $\begin{array}{l}\text { Carcharodus orientalis Reverdin, } \\
1913\end{array}$ & 2 (5.VI.2010), 3 (26.VIII.2009-19.IX.2015), 4 (8.VIII.2007, 24.VIII.2012) \\
\hline Spialia orbifer (Hübner, [1823]) & $\begin{array}{l}3 \text { (26.VIII.2009-8.VIII.2014), } 4 \text { (8.VIII.2007-21.VIII.2014-29.V.2015), } \\
7 \text { (3.V.2013), } 10 \text { (13.VIII.2001), } 11 \text { (28.IV.2008, 6.VI.2010, 20.VIII.2014), } \\
14 \text { (11.VIII.2005), } 19 \text { (7.V.2015), } 22 \text { (7.V.2015) }\end{array}$ \\
\hline
\end{tabular}




\begin{tabular}{|c|c|}
\hline Pyrgus malvae (Linnaeus, 1758) & $\begin{array}{l}3 \text { (3.V.2013), } 4 \text { (3.V.2008), } 11 \text { (28.IV.2008, 6.VI.2010), } 14 \text { (30.IV.2007), } \\
15 \text { (30.IV.2008), } 18 \text { (7.V.2015) }\end{array}$ \\
\hline $\begin{array}{l}\text { Pyrgus armoricanus (Oberthür, } \\
\text { 1910) }\end{array}$ & 3 (6.VI.2010), 4 (24.VIII.2012), 11 (17.VIII.2008) \\
\hline $\begin{array}{l}\text { Thymelicus acteon (Rottemburg, } \\
\text { 1775) }\end{array}$ & $\begin{array}{l}3 \text { (6.VI.2010, 28.VI.2013, 27.VI.2015), } 4 \text { (6.VII.2009), } 6 \text { (28.VI.2013), } \\
10 \text { (23.VI.2014), } 11 \text { (6.VI.2010), } 14 \text { (26.VI.2009-25.VI.2015), } \\
16 \text { (4.VII.2014, 29.V.2015) }\end{array}$ \\
\hline $\begin{array}{l}\text { Thymelicus lineola (Ochsenhe- } \\
\text { imer, 1808) }\end{array}$ & 3 (27.VI.2015), 10 (23.VI.2014) \\
\hline $\begin{array}{l}\text { Thymelicus sylvestris (Poda, } \\
\text { 1761) }\end{array}$ & 2 (5.VI.2010), 3 (6.VI.2010) \\
\hline Ochlodes sylvanus (Esper, [1777]) & $\begin{array}{l}2 \text { (5.VI.2010), } 3 \text { (6.VI.2010), } 4 \text { (29.V.2015), } 11 \text { (6.VI.2010), } \\
14 \text { (26.VI.2009- 25.VI.2015), } 16 \text { (29.V.2015) }\end{array}$ \\
\hline $\begin{array}{l}\text { Gegenes pumilio (Hoffmannsegg, } \\
\text { 1804) }\end{array}$ & $\begin{array}{l}2 \text { (13.IX.2002), } 4 \text { (8.VIII.2007, 19.VIII.2007), } 10 \text { (10.IX.2004), } \\
14 \text { (9.IX.2002), } 25 \text { (18.IX.2015) }\end{array}$ \\
\hline \multicolumn{2}{|l|}{ PAPILIONIDAE } \\
\hline $\begin{array}{l}\text { Iphiclides podalirius (Linnaeus, } \\
\text { 1758) }\end{array}$ & $\begin{array}{l}3 \text { (26.VIII.2009-27.VI.2015), } 4 \text { (3.V.2008, 6.VII.2009), } 7 \text { (3.V.2013, } \\
\text { 30.III.2014), } 9 \text { (27.IV.2008), } 10 \text { (13.VIII.2001- 18.VIII.2014), } \\
11 \text { (28.IV.2008), } 14 \text { (1.IX.1995-4.VIII.2015), } 20 \text { (7.V.2015), } \\
21 \text { (7.V.2015), } 24 \text { (4.V.2013) }\end{array}$ \\
\hline Papilio machaon (Linnaeus, 1758) & $\begin{array}{l}1 \text { (13.IX.2002), } 3 \text { (26.VIII.2009-19.IX.2015), } 7 \text { (3.V.2013, 30.III.2014), } \\
9 \text { (27.IV.2008), } 10 \text { (14.IX.2002- 20.VIII.2012), } 11 \text { (30.IV.2007-25.IV.2015), } \\
14 \text { (1.IX.1995- 4.VIII.2015), } 18 \text { (7.V.2015), } 19 \text { (7.V.2015), } 24 \text { (4.V.2013) }\end{array}$ \\
\hline $\begin{array}{l}\text { Zerynthia polyxena ([Denis \& } \\
\text { Schiffermüller], 1775) }\end{array}$ & $\begin{array}{l}3 \text { (3.V.2013), } 7 \text { (30.III.2014), } 9 \text { (27.IV.2008),11 (28.IV.2008, 25.IV.2015), } \\
14 \text { (27.IV.2007) }\end{array}$ \\
\hline \multicolumn{2}{|l|}{ PIERIDAE } \\
\hline Leptidea sinapis/juvernica & $\begin{array}{l}1 \text { (13.IX.2002), } 2 \text { (13.IX.2002), } 3 \text { (26.VIII.2009, 8.VIII.2014), } \\
4 \text { (8.VIII.2007-26.VIII.2009), } 9 \text { (27.IV.2008), } 10 \text { (13.VIII.2001-22. } \\
\text { VIII.2014), } 11 \text { (28.IV.2008, 17.VIII.2008, 6.VI.2010), } 14 \text { (11.VIII.2001- } \\
\text { 18.IX.2015), } 15 \text { (30.IV.2008), } 19 \text { (7.V.2015), } 20 \text { (7.V.2015), } 21 \text { (7.V.2015), } \\
22 \text { (7.V.2015), } 23 \text { (7.V.2015) }\end{array}$ \\
\hline $\begin{array}{l}\text { Anthocharis cardamines } \\
\text { (Linnaeus, 1758) }\end{array}$ & $\begin{array}{l}3 \text { (3.V.2013), } 4 \text { (3.V.2013), } 7 \text { (3.V.2013), } 11 \text { (30.IV.2007, 28.IV.2008), } \\
14 \text { (29.III.2014-25.IV.2015) } 18 \text { (7.V.2015), } 23 \text { (7.V.2015) }\end{array}$ \\
\hline Pieris brassicae (Linnaeus, 1758) & $\begin{array}{l}2 \text { (5.VI.2010), } 3 \text { (28.VI.2013), } 7 \text { (3.V.2013, 30.III.2014), } 10 \text { (13. } \\
\text { VIII.2001, 18.VIII.2014), 14 (2.VIII.1998- 27.IV.2014), } 20 \text { (7.V.2015) }\end{array}$ \\
\hline Pieris ergane (Geyer, 1828) & $\begin{array}{l}2 \text { (13.IX.2002, 5.VI.2010, 20.VIII.2013), } 3 \text { (26.VIII.2009- 19.IX.2015), } \\
4 \text { (3.V.2008, 6.VII.2009), } 9 \text { (27.IV.2008), } 16 \text { (4.VII.2014) }\end{array}$ \\
\hline Pieris mannii (Mayer, 1851) & $\begin{array}{l}2 \text { (5.VI.2010, 20.VIII.2013), } 3 \text { (26.VIII.2009, 6.VI.2010, 3.V.2013), } \\
4 \text { (6.VII.2009, 26.VIII.2009, 24.VIII.2012), } 7 \text { (3.V.2013, 21.VIII.2014), } \\
10 \text { (14.IX.2002, 15.VIII.2007, 22.VIII.2011), } 14 \text { (7.VIII.2014) }\end{array}$ \\
\hline Pieris rapae (Linnaeus, 1758) & $\begin{array}{l}1 \text { (13.IX.2002), } 2 \text { (13.IX.2002, 20.VIII.2013), } 3 \text { (26.VIII.2009, 3.V.2013, } \\
\text { 19.IX.2015), } 4 \text { (3.V.2008, 29.V.2015), } 7 \text { (3.V.2013, 30.III.2014), } \\
\text { 9 (27.IV.2008), } 10 \text { (13.VIII.2001-18.VIII.2014), } 11 \text { (28.IV.2008-25. } \\
\text { IV.2015), } 14 \text { (1.IX.1995-18.IX.2015), } 21 \text { (7.V.2015), } 25 \text { (18.IX.2015) }\end{array}$ \\
\hline Pontia edusa (Fabricius, 1777) & $\begin{array}{l}1 \text { (13.IX.2002), } 2 \text { (13.IX.2002), } 3 \text { (3.V.2013, 28.VI.2013, 19.IX.2015), } \\
4 \text { (19.VIII.2007, 16.VIII.2008), } 7 \text { (3.V.2013, 30.III.2014), } 9 \text { (27.IV.2008), } \\
10 \text { (14.IX.2002-20.VIII.2012), } 11 \text { (17.VIII.2008), } \\
14 \text { (9.IX.2002-7.VIII.2014), } 24 \text { (4.V.2013), } 25 \text { (18.IX.2015) }\end{array}$ \\
\hline Euchloe ausonia (Hübner, [1804]) & 7 (30.III.2014) \\
\hline
\end{tabular}




\begin{tabular}{|c|c|}
\hline Colias alfacariensis (Ribbe, 1905) & $\begin{array}{l}3 \text { (26.VIII.2009, 27.VI.2015, 19.IX.2015), } 4 \text { (3.V.2008, 16.VIII.2008), } \\
\text { 9 (27.IV.2008), } 10 \text { (13.VIII.2001, 14.IX.2002), 11 (28.IV.2008, } \\
\text { 17.VIII.2008, 25.IV.2015), 12 (17.VIII.2011), 13 (1.V.2007), } 18 \text { (7.V.2015), } \\
\text { 19 (7.V.2015), } 20 \text { (7.V.2015), } 21 \text { (7.V.2015), } 22 \text { (7.V.2015), } 23 \text { (7.V.2015), } \\
\text { 25 (18.IX.2015) }\end{array}$ \\
\hline Colias croceus (Fourcroy 1785) & $\begin{array}{l}\text { 1 (13.IX.2002), } 2 \text { (13.IX.2002, 5.VI.2010), } 3 \text { (26.VIII.2009- 19.IX.2015), } \\
\text { 4 (3.V.2008-21.VIII.2014), } 7 \text { (3.V.2013, 30.III.2014), } 9 \text { (27.IV.2008), 10 } \\
\text { (13.VIII.2001-18.VIII.2014), 11 (30.IV.2007-25.IV.2015), } 12 \text { (17. } \\
\text { VIII.2011), 13 (1.V.2007), 14 (13.VII.2001-18.IX.2015), 15 (30.IV.2008), } \\
\text { 17 (4.VII.2014), } 20 \text { (7.V.2015), } 22 \text { (7.V.2015), } 23 \text { (7.V.2015), } 24 \\
\text { (4.V.2013), 25 (18.IX.2015) }\end{array}$ \\
\hline $\begin{array}{l}\text { Gonepteryx cleopatra (Linnaeus, } \\
\text { 1767) }\end{array}$ & $\begin{array}{l}1 \text { (13.IX.2002), } 2 \text { (5.VI.2010), } 9 \text { (27.IV.2008), } 10 \text { (30.VI.2009, 23.VI.2014), } \\
\text { 14 (11.VIII.2005-4.VIII.2015) }\end{array}$ \\
\hline $\begin{array}{l}\text { Gonepteryx rhamni (Linnaeus, } \\
1758 \text { ) }\end{array}$ & 3 (6.VI.2010), 4 (3.V.2008) \\
\hline \multicolumn{2}{|l|}{ LYCAENIDAE } \\
\hline Lycaena phlaeas (Linnaeus, 1761) & $\begin{array}{l}1 \text { (13.IX.2002), } 2 \text { (5.VI.2010, 20.VIII.2013), } 3 \text { (26.VIII.2009-19.IX.2015), } \\
\text { 4 (8.VIII.2007-21.VIII.2014), } 7 \text { (3.V.2013, 30.III.2014, 21.VIII.2014), } \\
\text { 9 (27.IV.2008), } 10 \text { (13.VIII.2001-18.VIII.2014), 11 (28.IV.2008, } \\
\text { 17.VIII.2008), } 14 \text { (2.VIII.1998-18.IX.2015), } 18 \text { (7.V.2015), } 24 \text { (4.V.2013), } \\
\text { 25 (18.IX.2015) }\end{array}$ \\
\hline Callophrys rubi (Linnaeus, 1758) & 9 (27.IV.2008), 14 (29.III.2014, 27.IV.2014), 18 (7.V.2015), 23 (7.V.2015) \\
\hline Satyrium ilicis (Esper, 1779) & 2 (5.VI.2010), 3 (6.VI.2010, 28.VI.2013) \\
\hline $\begin{array}{l}\text { Satyrum spini ([Denis \& } \\
\text { Schiffermüller], 1775) }\end{array}$ & 2 (5.VI.2010), 3 (6.VI.2010), 4 (29.V.2015), 11 (6.VI.2010) \\
\hline Cacyreus marshalli Butler, 1898 & 10 (18. VIII.2014), 14 (22.VIII.2011-18.IX.2015) \\
\hline $\begin{array}{l}\text { Lampides boeticus (Linnaeus, } \\
\text { 1767) }\end{array}$ & 10 (14.IX.2002), 14 (12.VIII.2001, 12.IX.2002) \\
\hline $\begin{array}{l}\text { Leptotes pirithous (Linnaeus, } \\
\text { 1767) }\end{array}$ & $\begin{array}{l}2 \text { (13.IX.2002, 10.IX.2004), } 10 \text { (14.IX.2002), } 11 \text { (17.VIII.2008, } \\
\text { 20.VIII.2014), } 12 \text { (17.VIII.2011), } 14 \text { (12.IX.2002, 7.VIII.2014) }\end{array}$ \\
\hline Tarucus balkanica (Freyer, 1793) & $\begin{array}{l}3 \text { (26.VIII.2009, 3.V.2013, 19.IX.2015), } 4 \text { (19.VIII.2007), } 6 \text { (28.VI.2013), } \\
11 \text { (17.VIII.2008, 20.VIII.2014) }\end{array}$ \\
\hline Cupido minimus (Fuessly, 1775) & 3 (6.VI.2010, 28.VI.2013, 27.VI.2015), 4 (3.V.2008) \\
\hline $\begin{array}{l}\text { Pseudophilotes vicrama (Moore, } \\
\text { 1865) }\end{array}$ & 3 (6.VI.2010), 4 (3.V.2008) \\
\hline Scolitantides orion (Pallas, 1771) & 3 (6.VI.2010, 3.V.2013) \\
\hline $\begin{array}{l}\text { Celastrina argiolus (Linnaeus, } \\
1758 \text { ) }\end{array}$ & \begin{tabular}{|l|l|} 
2 (5.VI.2010), 3(6.VI.2010, 28.VI.2013), 4 (21.VIII.2014), 10 \\
(14.VIII.2001, 14.IX.2002), 11 (17.VIII.2008), 14 (1.IX.1995- 21.VIII.2015)
\end{tabular} \\
\hline Glaucopsyche alexis (Poda, 1761) & $\begin{array}{l}4 \text { (3.V.2008), } 9 \text { (27.IV.2008), } 11 \text { (25.IV.2015), } 13 \text { (1.V.2007), } 14 \\
\text { (30.IV.2007-25.IV.2015), } 18 \text { (7.V.2015), } 19 \text { (7.V.2015), } 20 \text { (7.V.2015), } \\
21 \text { (7.V.2015), } 23 \text { (7.V.2015) }\end{array}$ \\
\hline Plebejus argus (Linnaeus, 1758) & 16 (4.VII.2014, 29.V.2015), 20 (7.V.2015) \\
\hline Plebejus idas (Linnaeus, 1761) & 2 (5.VI.2010), 3 (6.VI.2010) \\
\hline $\begin{array}{l}\text { Aricia agestis ([Denis \& } \\
\text { Schiffermüller], 1775) }\end{array}$ & \begin{tabular}{|l} 
1 (13.IX.2002), 2 (5.VI.2010), 3 (6.VI.2010-19.IX.2015), 4 (8.VIII.2007- \\
24.VIII.2012), 5 (28.VI.2013), 6 (28.VI.2013), 7 (3.V.2013-25.IV.2015), 10 \\
(10.VIII.2011, 23.VI. 2014), 11 (30.IV.2007), 14 (1.IX.1995-23.VI. 2014), \\
17 (4.VII.2014), 19 (7.V.2015), 21 (7.V.2015), 23 (7.V.2015), 24 (4.V.2013)
\end{tabular} \\
\hline $\begin{array}{l}\text { Polyommatus escheri (Hübner, } \\
{[1823] \text { ) }}\end{array}$ & 3 (6.VI.2010), 4 (29.V.2015) \\
\hline
\end{tabular}




\begin{tabular}{|c|c|}
\hline $\begin{array}{l}\text { Polyommatus dorylas ([Denis \& } \\
\text { Schiffermüller], 1775) }\end{array}$ & 14 (1.IX.1995) \\
\hline $\begin{array}{l}\text { Polyommatus thersites (Cantener, } \\
\text { 1835) }\end{array}$ & 3 (6.VI.2010) \\
\hline $\begin{array}{l}\text { Polyommatus icarus (Rottem- } \\
\text { burg, 1775) }\end{array}$ & $\begin{array}{l}1 \text { (13.IX.2002), } 2 \text { (13.IX.2002, 5.VI.2010), } 3 \text { (26.VIII.2009- 19.IX.2015), } \\
4 \text { (8.VIII.2007- 21.VIII.2014, 29.V.2015), } 5 \text { (28.VI.2013), } 6 \text { (28.VI.2013), } \\
7 \text { (3.V.2013, 21.VIII.2014), } 8 \text { (18.VIII.2008), } 9 \text { (27.IV.2008), } \\
10 \text { (14.IX.2002- 18.VIII.2014), } 11 \text { (30.IV.2007- 20.VIII.2014), } \\
12 \text { (17.VIII.2011), } 13 \text { (1.V.2007), } 14 \text { (1.IX.1995-25.VI.2015), } \\
15 \text { (30.IV.2008), } 16 \text { (4.VII.2014, 29.5.2015), } 17 \text { (4.VII.2014), } 18 \text { (7.V.2015), } \\
20 \text { (7.V.2015), } 23 \text { (7.V.2015), 24 (4.V.2013), } 25 \text { (18.IX.2015) }\end{array}$ \\
\hline $\begin{array}{l}\text { Polyommatus daphnis ([Denis \& } \\
\text { Schiffermüller], 1775) }\end{array}$ & 6 (28.VI.2013) \\
\hline $\begin{array}{l}\text { Polyommatus bellargus (Rottem- } \\
\text { burg, 1775) }\end{array}$ & $\begin{array}{l}1 \text { (13.IX.2002), } 2 \text { (5.VI.2010), } 3 \text { (26.VIII.2009-19.IX.2015), } \\
4 \text { (8.VIII.2007- 29.V.2015), } 7 \text { (3.V.2013, 21.VIII.2014), 9 (18.VIII.2008), } \\
10 \text { (13.VIII.2001- 18.VIII.2014), } 11 \text { (17.VIII.2008, 6.VI.2010, } \\
\text { 20.VIII.2014), } 12 \text { (17.VIII.2011), } 13 \text { (1.V.2007), } 14 \text { (1.IX.1995-18.IX.2015), } \\
16 \text { (29.V.2015), } 18 \text { (7.V.2015), } 25 \text { (18.IX.2015) }\end{array}$ \\
\hline \multicolumn{2}{|l|}{ NYMPHALIDAE } \\
\hline Libythea celtis (Laicharting 1782) & $\begin{array}{l}2 \text { (5.VI.2010), } 3 \text { (6.VI.2010, 3.V.2013, 19.IX.2015), } 4 \text { (29.V.2015), } \\
7 \text { (30.III.2014) }\end{array}$ \\
\hline $\begin{array}{l}\text { Argynnis pandora ([Denis \& } \\
\text { Schiffermüller], 1775) }\end{array}$ & $\begin{array}{l}1 \text { (13.IX.2002), } 2 \text { (5.VI.2010), } 3 \text { (6.VI.2010, 19.IX.2015), } 4 \text { (8.VIII.2007- } \\
\text { 21.VIII.2014), } 10 \text { (14.IX.2002), } 14 \text { (12.IX.2002-22.VIII.2011) }\end{array}$ \\
\hline Argynnis niobe (Linnaeus, 1758) & 3 (6.VI.2010, 27.VI.2015), 4 (6.VII.2009) \\
\hline Issoria lathonia (Linnaeus 1758) & $\begin{array}{l}3 \text { (3.V.2013), } 4 \text { (19.VIII.2007, 3.V.2008, 26.VIII.2009), } 7 \text { (30.III.2014), } \\
10 \text { (14.VIII.2001) }\end{array}$ \\
\hline Vanessa atalanta (Linnaeus, 1758) & $\begin{array}{l}3 \text { (6.VI.2010, 3.V.2013), } 6 \text { (28.VI.2013), } 9 \text { (27.IV.2008), } 10 \text { (14.VIII.2001, } \\
\text { 14.IX.2002), } 11 \text { (28.IV.2008, 17.VIII.2008), } 14 \text { (1.IX.1995-18.IX.2015) }\end{array}$ \\
\hline Vanessa cardui (Linnaeus, 1758) & $\begin{array}{l}1 \text { (13.IX.2002), } 3 \text { (26.VIII.2009-19.IX.2015), } 4 \text { (3.V.2008, 6.VII.2009), } \\
7 \text { (30.III.2014), } 9 \text { (27.IV.2008), } 10 \text { (13.VIII.2001, 14.IX.2002, 30.VI.2009), } \\
12 \text { (17.VIII.2011), } 14 \text { (1.IX.1995-7.VIII.2014), 18 (7.V.2015), } 20 \text { (7.V.2015), } \\
23 \text { (7.V.2015), } 24 \text { (4.V.2013), } 25 \text { (18.IX.2015) }\end{array}$ \\
\hline $\begin{array}{l}\text { Polygonia c-album (Linnaeus, } \\
1758 \text { ) }\end{array}$ & 3 (6.VI.2010) \\
\hline Polygonia egea (Cramer, 1775) & $\begin{array}{l}1 \text { (13.IX.2002), } 2 \text { (5.VI.2010), } 3 \text { (6.VI.2010-19.IX.2015), } 4 \text { (8.VIII.2007- } \\
\text { 26.VIII.2009) }\end{array}$ \\
\hline $\begin{array}{l}\text { Nymphalis polychloros (Linnaeus, } \\
1758)\end{array}$ & 3 (6.VI.2010), 7 (30.III.2014), 14 (29.III.2014) \\
\hline $\begin{array}{l}\text { Euphydryas aurinia (Rottemburg, } \\
1775 \text { ) }\end{array}$ & $\begin{array}{l}3 \text { (6.VI.2010), } 9 \text { (27.IV.2008), } 11 \text { (30.IV.2007, 28.IV.2008), } \\
15 \text { (30.IV.2008), } 18 \text { (7.V.2015), } 20 \text { (7.V.2015), } 21 \text { (7.V.2015), } \\
22 \text { (7.V.2015), } 24 \text { (4.V.2013) }\end{array}$ \\
\hline Melitaea cinxia (Linnaeus, 1758) & $\begin{array}{l}3 \text { (6.VI.2010, 3.V.2013), } 4 \text { (3.V.2008), } 9 \text { (27.IV.2008),11 (30.IV.2007, } \\
\text { 28.IV.2008), } 14 \text { (27.IV.2007, 27.IV.2008), } 16 \text { (29.V.2015), } 18 \text { (7.V.2015), } \\
19 \text { (7.V.2015), } 22 \text { (7.V.2015) }\end{array}$ \\
\hline Melitaea didyma (Esper, 1778) & $\begin{array}{l}3 \text { (26.VIII.2009-27.VI.2015), } 4 \text { (8.VIII.2007, 16.VIII.2008, 26.VIII.2009), } \\
7 \text { (21.VIII.2014), } 8 \text { (18.VIII.2008),10 (13.VIII.2001-18.VIII.2014), } \\
11 \text { (17.VIII.2008, 20.VIII.2014), } 12 \text { (17.VIII.2011), } \\
14 \text { (1.IX.1995-4.VIII.2015), } 17 \text { (4.VII.2014) }\end{array}$ \\
\hline Melitaea ornata Christoph, 1893 & 3 (3.V.2013), 7 (3.V.2013), 24 (4.V.2013) \\
\hline $\begin{array}{l}\text { Melitaea phoebe ([Denis \& } \\
\text { Schiffermüller], 1775) }\end{array}$ & 3 (3.V.2013), 24 (4.V.2013) \\
\hline
\end{tabular}




\begin{tabular}{|c|c|}
\hline Charaxes jasius (Linnaeus, 1767) & $\begin{array}{l}9 \text { (8.VIII.2008), } 10 \text { (13.IX.1997-18.VIII.2014), } \\
14 \text { (13.VIII.2001-18.IX.2015) }\end{array}$ \\
\hline $\begin{array}{l}\text { Limenitis reducta (Staudinger, } \\
\text { 1901) }\end{array}$ & $\begin{array}{l}1 \text { (13.IX.2002), } 3 \text { (26.VIII.2009-19.IX.2015), } 4 \text { (8.VIII.2007-29.V.2015), } \\
6 \text { (28.VI.2013), } 10 \text { (13.VIII.2001-18.VIII.2014), } 16 \text { (29.V.2015) }\end{array}$ \\
\hline Pararge aegeria (Linnaeus, 1758) & $\begin{array}{l}3 \text { (27.VI.2015), } 4 \text { (3.V.2008, 21.VIII.2014), } 5 \text { (28.VI.2013), } \\
9 \text { (27.IV.2008), } 10 \text { (14.IX.2002), } 14 \text { (1.IX.1995- 18.IX.2015), } \\
21 \text { (7.V.2015) }\end{array}$ \\
\hline $\begin{array}{l}\text { Lasiommata } m \\
1758)\end{array}$ & 4 (8.VIII.2007-21.VIII.2014), 14 (1.IX.1995) \\
\hline $\begin{array}{l}\text { Lasiommata megera (Linnaeus, } \\
1767)\end{array}$ & $\begin{array}{l}2 \text { (13.IX.2002, 10.IX.2004), } 3 \text { (3.V.2013, 28.VI.2013, 27.VI.2015), } 4 \\
\text { (3.V.2008, 6.VII.2009, 21.VIII.2014), } 5 \text { (28.VI.2013), } 6 \text { (28.VI.2013), } 7 \\
\text { (3.V.2013, 30.III.2014, 21.VIII.2014), } 9 \text { (27.IV.2008),10 (13.VIII.2001- } \\
\text { 26.VI.2014), } 11 \text { (30.IV.2007-25.IV.2015), } 12 \text { (17.VIII.2011), 13 } \\
\text { (1.V.2007), } 14 \text { (1.IX.1995- 18.IX.2015), } 15 \text { (30.IV.2008), 16 (4.VII.2014), } \\
\text { 17 (4.VII.2014), } 18 \text { (7.V.2015), } 19 \text { (7.V.2015), } 20 \text { (7.V.2015), } 22 \\
\text { (7.V.2015), } 23 \text { (7.V.2015), 24 (4.V.2013), 25 (18.IX.2015) }\end{array}$ \\
\hline $\begin{array}{l}\text { Coenonympha pamp } \\
\text { (Linnaeus, 1758) }\end{array}$ & $\begin{array}{l}1 \text { (13.IX.2002), } 2 \text { (13.IX.2002), } 3 \text { (26.VIII.2009- 19.IX.2015), } \\
4 \text { (8.VIII.2007, 29.V.2015), } 7 \text { (3.V.2013, 21.VIII.2014, 25.IV.2015), } \\
8 \text { (18.VIII.2008), } 9 \text { (27.IV.2008), } 10 \text { (13.VIII.2001-18.VIII.2014), } \\
11 \text { (28.IV.2008- 25.IV.2015), } 12 \text { (17.VIII.2011), 13 (1.V.2007), } \\
14 \text { (2.VIII.1998-18.IX.2015), } 15 \text { (30.IV.2008), } 16 \text { (4.VII.2014, 29.V.2015), } \\
18 \text { (7.V.2015), } 19 \text { (7.V.2015), } 20 \text { (7.V.2015), } 21 \text { (7.V.2015), } 22 \text { (7.V.2015), } \\
23 \text { (7.V.2015), } 24 \text { (4.V.2013), } 25 \text { (18.IX.2015) }\end{array}$ \\
\hline Pyronia cecilia (Valla & $\begin{array}{l}3 \text { (28.VI.2013, 8.VIII.2014, 19.IX.2015), } 4 \text { (8.VIII.2007), } 5 \text { (28.VI.2013), } \\
6 \text { (28.VI.2013), } 7 \text { (21.VIII.2014), } 11 \text { (17.VIII.2008, 20.VIII.2014), } 14 \text { (2. } \\
\text { VIII.1998-7.VIII.2014), } 17 \text { (4.VII.2014) }\end{array}$ \\
\hline Pyronia tithonus (Linnaeus, 1767) & 14 (5.VII.2011) \\
\hline Maniola jur & $\begin{array}{l}1 \text { (13.IX.2002), } 2 \text { (13.IX.2002-19.IX.2015), } 3 \text { (26.VIII.2009, 6.VI.2010, } \\
\text { 28.VI.2013), } 4 \text { (8.VIII.2007, 29.V.2015), } 5 \text { (28.VI.2013), } 6 \text { (28.VI.2013), } \\
7 \text { (21.VIII.2014), } 8 \text { (18.VIII.2008), } 10 \text { (15.VIII.2007-23.VI.2014), } \\
11 \text { (17.VIII.2008, 6.VI.2010, 20.VIII.2014), } 14 \text { (1.IX.1995- 18.IX.2015), } \\
16 \text { (4.VII.2014, 29.V.2015), } 25 \text { (18.IX.2015) }\end{array}$ \\
\hline Hyponephele lupina (Costa, 1836) & 4 (8.VIII.2007, 19.VIII.2007) \\
\hline Proterebia afra (Fabricius, 1787) & $\begin{array}{l}3 \text { (3.V.2013), } 4 \text { (3.V.2008), } 6 \text { (3.V.2013), } 7 \text { (3.V.2013, 25.IV.2015), } \\
23 \text { (7.V.2015) }\end{array}$ \\
\hline $\begin{array}{l}\text { Melanargia galathea (Linnaeus, } \\
1758 \text { ) }\end{array}$ & $\begin{array}{l}4 \text { (6.VII.2009), } 5 \text { (28.VI.2013), } 10 \text { (30.VI.2009), } 11 \text { (6.VI.2010), } \\
14 \text { (26.VI.2009-25.VI.2015), } 16 \text { (4.V.2014, 29.V.2015), } 17 \text { (4.VII.2014) }\end{array}$ \\
\hline Melanargia larissa (Geyer, 1828) & 3 (6.VI.2010) \\
\hline $\begin{array}{l}\text { Hipparchia semele (Linnaeus, } \\
\text { 1758) }\end{array}$ & $\begin{array}{l}1 \text { (13.IX.2002), } 2 \text { (13.IX.2002), } 3 \text { (28.VI.2013-19.IX.2015), } \\
4 \text { (8.VIII.2007, 6.VII.2009, 24.VIII.2012), } 5 \text { (28.VI.2013), } 6 \text { (28.VI.2013), } \\
10 \text { (14.VIII.2001-18.VIII.2014), } 14 \text { (1.IX.1995-29.VI.2013) }\end{array}$ \\
\hline $\begin{array}{l}\text { Hipparchia statilinus (Hufnagel, } \\
\text { 1766) }\end{array}$ & $\begin{array}{l}1 \text { (13.IX.2002), } 2 \text { (13.IX.2002- 20.VIII.2013), } 3 \text { (26.VIII.2009, } \\
\text { 19.IX.2015), } 4 \text { (8.VIII.2007-21.VIII.2014), } 7 \text { (21.VIII.2014), } \\
8 \text { (18.VIII.2008), } 10 \text { (13.VIII.2001- 20.VIII.2012), } 11 \text { (17.VIII.2008, } \\
\text { 20.VIII.2014), } 12 \text { (17.VIII.2011), } 14 \text { (1.IX.1995-14.VIII.2014) }\end{array}$ \\
\hline $\begin{array}{l}\text { Hipparchia syriaca (Staudinger, } \\
1871 \text { ) }\end{array}$ & $\begin{array}{l}1 \text { (13.IX.2002), } 3 \text { (28.VI.2013-19.IX.2015), } 4 \text { (24.VIII.2012), } 5 \text { (28.VI.2013), } \\
7 \text { (21.VIII.2014) }\end{array}$ \\
\hline Brintesia circe (Fabricius, 1775) & $\begin{array}{l}3 \text { (28.VI.2013, 19.IX.2015), } 4 \text { (16.VIII.2008, 26.VIII.2009, 24.VIII.2012), } \\
16 \text { (4.VII.2014), } 17 \text { (4.VII.2014) }\end{array}$ \\
\hline Chazara briseis (Linnaeus, 1764) & $\begin{array}{l}3 \text { (27.VI.2015), } 4 \text { (26.VIII.2009), } 10 \text { (13.VIII.2001, 14.IX.2002), } \\
17 \text { (4.VII.2014) }\end{array}$ \\
\hline
\end{tabular}


Among the species listed several could be considered common and widespread in northern Dalmatia (observed at $75 \%$ of the sites): Colias croceus, Polyommatus icarus, Lasiommata megera, and Coenonympha pamphilus. On the other hand several species were observed only at a single location and could be considered extremely rare in the region: Euchloe ausonia, Scolitantides orion, Polyommatus dorylas, Polyommatus thersites, Polyommatus daphnis, Polygonia c-album, Melitaea phoebe, Pyronia tithonus, Hyponephele lupina, and Melanargia larissa. It is important to note that the majority of these species were observed on limestone outcrops neighbouring the Karin and Novigrad Sea where several small gullies and valleys represent the most butterfly-rich habitats in the region.

According to the Red Data List of Croatian Butterflies (Šsšić et al., 2013) eight species among those observed are considered near threatened (NT): Papilio machaon, Zerynthia polyxena, Glaucopsyche alexis, Polyommatus thersites, Pseudophilotes vicrama, Scolitantides orion, Euphydryas aurinia, and Proterebia afra. Thymelicus acteon and Pieris brassicae are considered data deficient (DD). On the European level there are five species considered near threatened (NT) in the region: Carcharodus floccifera, Thymelicus acteon, Polyommatus dorylas, Pseudophilotes vicrama, and Chazara briseis. Only Euphydryas aurinia is listed on the Habitats Directive Annexes II and IV (Anonymus, 1992).

The highest diversity was recorded in the Bijelina Valley (64 species) and in nearby Karišnica Valley (53). The Batalaža Peninsula at Privlaka has the third largest recorded diversity (46 species), but has been oversampled compared to other localities as it is the summer residence of the first and the last author. In terms of presence of threatened species Bijelina Valley stands out with 11 recorded species (Fig. 2).

\section{DISCUSSION}

Compared to neighbouring regions, in particularly Zrmanja River (104 species) and Velebit Mts. (137 species), northern Dalmatia has a more depauperate butterfly fauna

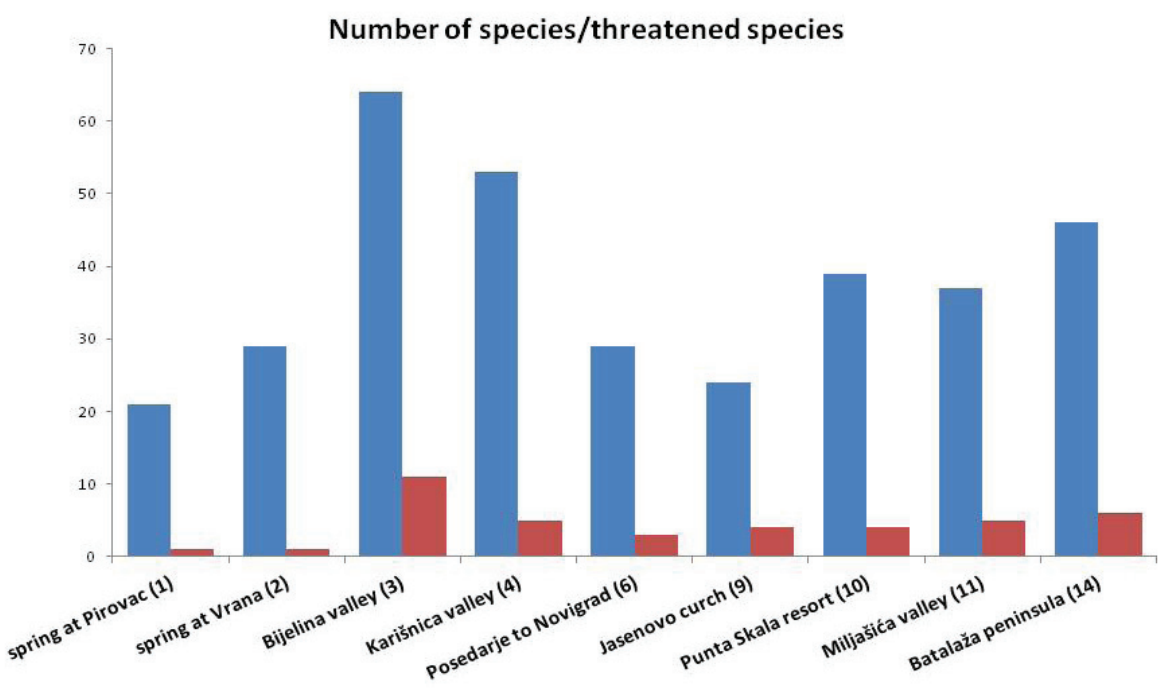

Fig. 2. Butterfly diversity and distribution of threatened species at ten most species rich localities in northern Dalmatia. 


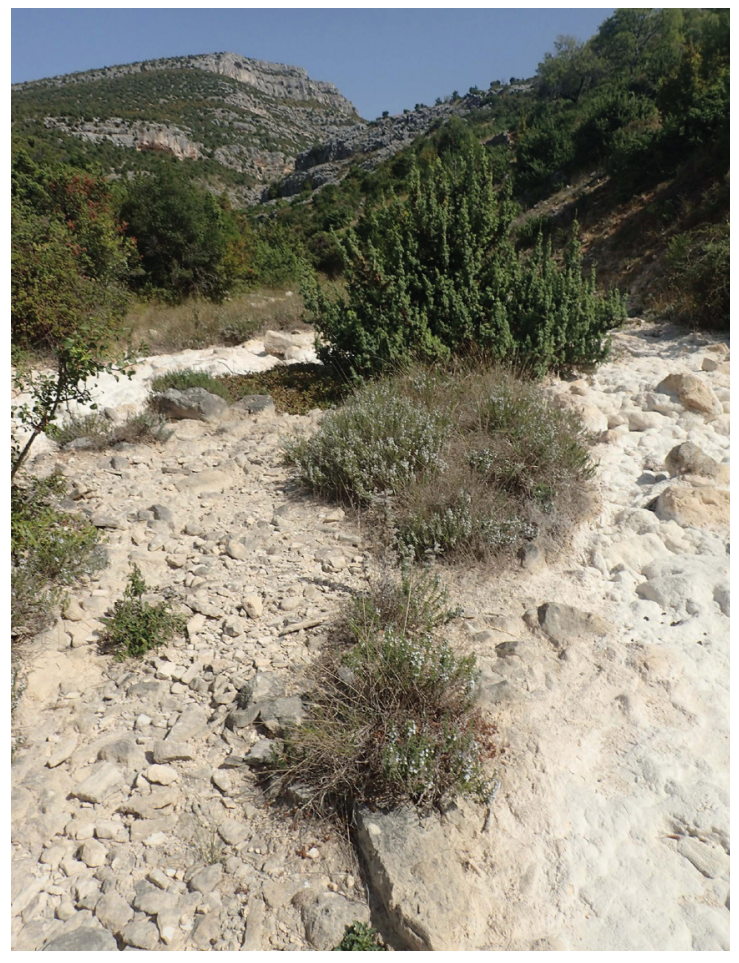

Fig. 3. Typical habitat in Bijelina Valley with dry riverbed and abundance of flowering thymus where butterflies congregate in late summer and autumn.

(Minoci et al., 2007; Koren et al., 2011). However, the fauna is still richer than on the neighbouring large islands: Pag (54 species), Pašman (50 species), Ugljan (43 species), and Dugi otok (47 species) (Withrington \& VerovNiK, 2008; Verovnik, 2011). Due to a lack of historical records it is hard to say whether some species have become extinct in the surveyed region. HABELER (1976) lists the following species for the region that were not recorded during our survey: Carcharodus lavatherae (Esper, [1783]), Pyrgus sidae (Esper, [1784]), Cupido alcetas (Hoffmannsegg, 1804), Nymphalis antiopa (Linnaeus, 1758), Argynnis adippe ([Denis \& Schiffermüller], 1775). The majority of these species were observed further south from Vrana Lake and Pirovac, beyond the surveyed region. The latter two species are likely to be observed in the more wooded Karin region, while Pyrgus sidae is extremely rare in Croatia with recent records only from the mountains in the Dalmatian hinterland (Tvrtкović et al., 2012; Koren \& LaUš,

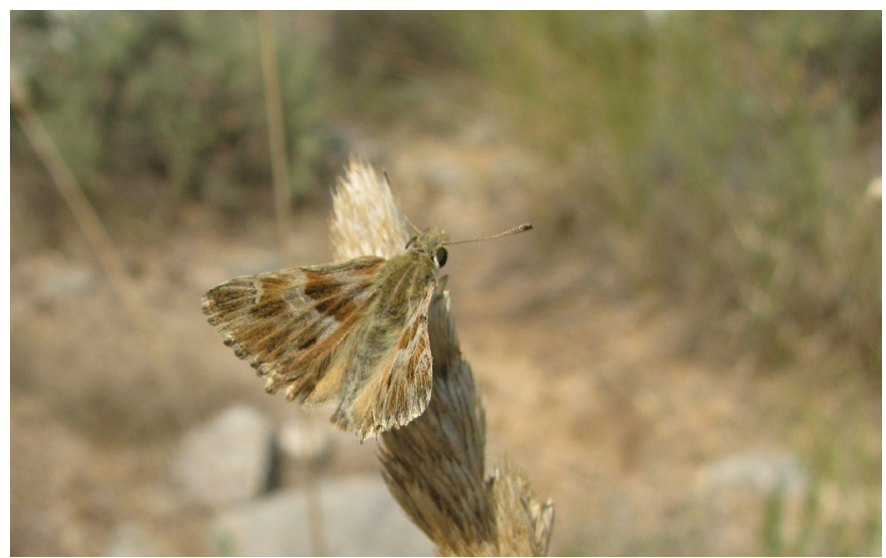

Fig. 4. Male of The Oriental Skipper (Carcharodus orientalis) typically perched on dry grass stem in Bijelina Valley. 


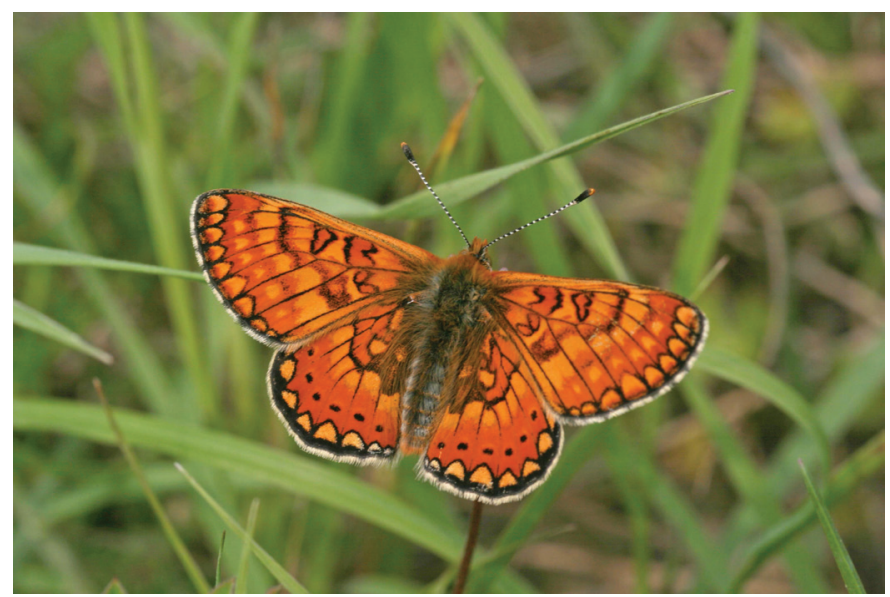

Fig. 5. The Marsh Fritilary (Euphydryas aurinia) is very brightly coloured in northern Dalmatia.

2013; Koren et al., 2015b) and the Mt Biokovo (Minoci et al., 2011). Cupido alcetas is considered widespread in Dalmatia, however no exact observation localities exist (LoRKović, 2009), and it has not been observed in any recent studies in the region.

Among the species observed, several are rare in Croatia or reach their northern limit of distribution in the surveyed region and require further comments:

\section{Carcharodus orientalis Reverdin, 1913}

Due to its similarity to Carcharodus floccifera, not much is known about its distribution in Croatia. Differences in male genitalia and the distinctive tuft of dark hair on the forewing underside are useful for identification (LAFRANCHIS, 2004; TOLMAN \& LEWINGTON, 2008). It was first mentioned for Croatia by STAUDER (1923) for the surroundings of Split as Carcharodus baeticus (Rambur, 1842). Although listed as present in Croatia (LoRKović, 2009; ŠAšić et al., 2011) recent records of this species in Croatia are very scarce, with only a single recent record, from the island Vis (Withrington \& VERovniK, 2008). We have observed the species repeatedly in Karišnica and Bijelina Valley near Karin where it possibly flies in three generations with fresh males observed also in September. We found the species once also at a strong spring E of the Vrana village on wet sands along the stream. The butterflies seem to prefer hot rocky sheltered places and males are typically territorial, perching on dry stems along dry riverbeds or paths. At Bijelina valley the species co-occurs with its close relative $C$. floccifera. It is difficult to judge if these records represent the northern limit of $C$. orientalis distribution along the Adriatic coast, as historical records also exist from the northernmost Adriatic, from Trieste (STAUDER, 1923).

\section{Gegenes pumilio (Hoffmannsegg, 1804)}

Another rare species in Dalmatia with only a handful of published records (LoRKović, 1971; VerovniK, 2011; Koren et al., 2015a). Until Lorković's study all Gegenes records were automatically assigned to the closely related G. nostrodamus (Fabricius, 1793) (STAUdER, 1923; BuRgERMEISTER, 1964) including the record form Pag Island (MLAdinov, 1965); however, this species is extremely rare in Dalmatia and possibly limited to the souther- 
nmost part of Croatia (LoRković, 1971). Our observations are in line with records from the neighbouring islands of Vir (Koren et al., 2015a) and Pašman (VERovniK, 2011) where the species was recorded at singular sites. The species was common in the middle part of the Karišnica valley in August 2007, but otherwise only single specimens were observed at Vrana, Petrčani and Privlaka. This implies that the species is only an occasional vagrant in northern Dalmatia with no permanent colonies. The currently known northern limit of the species is at Mala Paklenica Valley at Starigrad where we found it in August 2007. It is possible that the record of G. nostrodamus from Pag Island (MLAdinov, 1967) also refers to G. pumilio, as it was recorded at the time when G. pumilio was not considered present in Dalmatia (LoRKović, 1971).

\section{Euchloe ausonia (Hübner, [1804])}

The species reaches its northern limit of distribution on Pag Island (ZАKšEK, 2005). It has also been recorded at two sites along the Zrmanja River (Koren et al., 2011), so the new locality at Posedarje on the shores of Novigrad Sea provides a link between the two known distribution regions.

\section{Cacyreus marshalli Butler, 1898}

This non-native butterfly species only recently invaded the Croatian coast (VEROVNIK \& Kosmač, 2009) and has been recorded also from several islands (Kučinić et al., 2013; Koren et al., 2014; Koren et al., 2015a). In northern Dalmatia it was first observed in Privlaka on geraniums in gardens in 2011. In 2014 a single specimen was found in maquis bordering Punta Skala resort near Petrčani. Despite the general trend of expansion in Croatia the species is by no means widespread or common in northern Dalmatia.

\section{Tarucus balkanica (Freyer, 1793)}

According to historical records the species was known from northern Dalmatia in Zadar and Biograd (Mladinov, 1973), Pakoštane (Habeler, 1976) and also further north at Starigrad (LoRKović, 2009). We confirm the latter record, as several adults have been observed along the trail in Mala Paklenica Valley in August 2007 and 2010. In northern Dalmatia the species is confined to limestone areas and it flies in sheltered rocky gullies. In Bijelina Valley near Karin the species can be numerous in August, whereas in other localities (Karišnica Valley, Miljašića Valley, Novigrad) only single specimens were observed. The species was recently found also in the neighbouring Zrmanja River region (Koren et al., 2011).

\section{Polyommatus thersites (Cantener, 1835)}

The species is rare in Croatia (Minoci \& ŠAšıć, 2006) with few records also from the Dalmatian hinterland (Hafner, 1994, Tvrtкović et al., 2012) and in the Zrmanja valley (Koren et al., 2011). So far only two males were observed in the Bijelina Valley near Karin in June 2010, comprising the only records for the species in Northern Dalmatia.

\section{Euphydryas aurinia (Rottemburg, 1775)}

This is the only Habitats Directive species (AnON., 1992) present in the surveyed region. It has been known from Zadar from the material in the collection of the Croatian national zoological museum in Zagreb (MLadinov, 1973), the record confirmed also by 
HABELER (1976). Recently there are several records from the surroundings of Benkovac, and the area is included in the Natura 2000 site Benkovac (HR2001218) (ŠAšIĆ-KLJAJO \& Minoci, 2009). The adults from this region are large and brightly coloured possibly belonging to f. provincialis (STAUDER, 1922). Although we found the species in large numbers in wet meadows and neighbouring dry grasslands with rich flowering in Miljašića Valley, no specimens were seen after 2008. The evident decline was possibly caused by abandonment and overgrowth of the meadows. Since it is still common and abundant at localities near Ljubač, Vrsi and Žerava, this part of northern Dalmatia could qualify as a Natura 2000 site for the species. The species was found also on Vir Island just across the bridge from the mainland locality (Koren et al., 2015).

\section{Hyponephele lupina (Costa, 1836)}

Due to its similarity with the more widespread Hyponephele lycaon Rottemburg, 1775 this species was commonly overlooked in the past surveys. However, both species are rare in Croatia with $\mathrm{H}$. lycaon more widely distributed at higher altitudes (Mrнос et al., 2007; Lorković, 2009; TvrTKović et al., 2012). Only single females of the species were observed, on two occasions in August 2007 in Karišnica Valley, relatively close to the known localities in the Zrmanja Valley (Koren et al., 2011). Because the valley was already considerably overgrown at that time there is hardly any chance the species has survived at that locality.

\section{Proterebia afra (Fabricius, 1787)}

This is an emblematic species for the Dalmatian butterfly fauna which has been well studied in the last decade (Minoci \& ŠAšić, 2007; Koren et al., 2010). The species is much more widespread than was previously known and is reported to be common also from the Zrmanja Valley (Koren et al., 2011). We found it on slopes above the Novigrad Sea near Posedarje and at Jovići village which geographically closes the gap between the localities known in the Zrmanja River region and those from Pag Island (ZAKšEK, 2005). It was recorded also in the Karišnica and the Bijelina Valley near Karin, but in low densities. On 25.4.2015 we found the species at two additional sites north of Posedarje: 1. Posedarje, at the NW edge of the town near the highway exit; $44^{\circ} 13^{\prime} 13.88^{\prime \prime} \mathrm{N}, 15^{\circ} 27^{\prime} 12.36^{\prime \prime} \mathrm{E}$. 2. Gronja Slivnica, rocky pastures N of the village; $44^{\circ} 14^{\prime} 42.78^{\prime \prime} \mathrm{N}, 15^{\circ} 26^{\prime} 14.39^{\prime \prime} \mathrm{E}$.

\section{Melanargia larissa (Geyer, 1828)}

The species is locally distributed along most of the Croatian coast from Rijeka to Gruž, but with large gaps (STAUder, 1922; Lorković, 2009). The species is rarely mentioned in recent reports (TVRTKOvić et al., 2012; Koren et al., 2015b) and has not been recorded from the Velebit Mts in a recent study (Мiносл et al., 2007). It is however widespread on Krk Island further north (HABELER, 2003). We found it on rocky and grassy slopes along the Bijelina Valley in low densities. The species is not listed for the Zrmanja River region (Koren et al., 2011), but we did record it there at the Krupa spring in August 2015.

Although northern Dalmatia is not particularly important for butterfly conservation, there are some sites that host a large variety of species (Fig. 2), including several species listed in the national red list (ŠAšı́ et al., 2013). Such sites, suitable for butterfly micro-reserves, are the Bijelina and Karišnica valleys. These valleys and the majority of other visited localities are in urgent need of management, as abandonment resulting in the overgrowing of open grasslands is already reducing the butterfly diversity and is in particu- 
larly threatening to rare and local habitat specialist species. Another threat, more evident in coastal areas, is the destruction of habitats due to the building of infrastructure and the extension of urban areas. The only large stretch of maquis left in northern Dalmatia at Petrčani that has been recently reduced due to thed building of a tourist resort is a good example. We hope that our contribution will generate further studies in the region and provide additional background information for conservation of butterflies in Dalmatia.

\section{ACKNOWLEDGMENTS}

We wish to express our gratitude to Stoyan Beshkov for providing additional literature regarding the butterfly fauna of Dalmatia.

Received September 25, 2015

\section{REFERENCES}

Abafi - Aigner, L. \& PÁvel, J., 1900: A Magyar biradolom állatvilága. III - Arthropoda. Kyrali Magiar Természettudományi Társulat. Budimpešta. pp 29-42.

Anon., 1992: Habitat Directive Annexes II and IV. Council Directive 92/43/EEC of 21 May 1992 on the conservation of natural habitats and wild fauna and flora. Annex I-VI. Council of the European Communities.

Buresch, I. \& Iltschew, D., 1915: Zweiter Beitrag zur Erforschung der Lepidopterenfauna von Trazien Mazedonien und der Nachbarländer. - Trav. Soc. Bulg. Sci. Natur. 8, 151-197. (In Bulgarian).

Bürgermeister, F., 1964: Makrolepidopteren aus dem räume Dubrovnik (Süddalmatien, FVR Jugoslavien). Z. Wiener Entomol. Ges. 49, 137-152.

Dincă, V., Lukhtanov, V. A., Talavera, G. \& Vila, R., 2011: Unexpected layers of cryptic diversity in wood white Leptidea butterflies. Nat. Commun. 2, 324.

Fauna Europata, 2015: Fauna Europaea version 2.5. Web Service available online at http://www.faunaeur.org (accessed on 10.2.2015).

Galvagni, E., 1902: Beiträge zur Kenntnis der fauna einiger dalmatischer inseln. Ver.Zool.-bot. Ges. Wien $52,362-380$.

GalvaGni, E., 1909: Die zoologische reise des naturwissenschaftlichen vereines nach Dalmatien im April 1906 (Beiträge zur Kenntnis der lepidopterenfauna der Adriatischen inseln). Sonder-Abdruck Mitt. Naturwiss. Verr. Univ. Wien 7, 154-254.

Germar, E., 1814: Reise durch Österreich und Tyrol nach Dalmatien und Ragusa. Brockhaus, Leipzig 1, $273-280$.

Habeler, H., 1976: Beitrag zur Lepidopterenfauna Dalmatiens. Acta Entomol. Jug. 12, 67-87.

Habeler, H., 2003: Die Schmetterlinge der Adria-Insel Krk. Eine ökofaunistische Studie. Entomol. Esperiana, Graz, 221 pp.

HAfNer, I., 1994: Verzeichnis der bei Knin gesammelten Schmetterlinge (Lepidoptera). Nat. Croat. 3(2), 119-184.

Koren, T. \& BJelić, M., 2010: New data about butterflies and moths (Insecta: Lepidoptera) of Kornati islands, Croatia. Entomol. Croat. 14(3/4), 45-52.

Koren, T., Burić, I., ŠTiH, A., ZAKŠEK, V. \& VerovniK, R., 2010: New data about the distribution and altitudinal span of the Dalmatian ringlet, Proterebia afra dalmata (Godart, /1824/) (Lepidoptera: Satyrinae) in Croatia. Acta Entomol. Slov. 18(2), 143-150.

Koren, T., BJelić, M., Božınovska, E., ŠTiH, A. \& Burić, I., 2011: Contribution to the knowledge of butterfly fauna (lepidoptera: rhopalocera) of Zrmanja river region Croatia. Acta Entomol. Slov. 19 (2), $155-168$.

Koren, T., Beretta, S., Črne, M. \& Verovnik, R., 2013a: On the distribution of Pyrgus malvoides (Elwes \& Edwards, 1897) (Lepidoptera: Hesperiidae) at the eastern part of its range. Entomol. Gaz. 64, 225-234.

Koren, T., Črne, M. \& Španić, R., 2013b: On the questionable record of the Balkan Grayling, Hipparchia senthes (Fruhstorfer, 1908), in Croatia (Lepidoptera: Nymphalidae, Satyrinae). Nachr. Entomol. Ver. Apollo 34(3), 133-136.

Koren, T. \& Lauš, B., 2013: Dinara Massif - a new hotspot for the butterfly (Papilionoidea) diversity of the Dinaric Arc. Nota Lepidopterol.36(2), 109-126. 
Konen, T. \& ŠTiн A., 2013: On the occurrence of Eastern knapweed fritillary, Melitaea ornata (Lepidoptera: Nymphalidae) in Croatia. Phegea 41(3), 63-66.

Koren, T., BJelić, M., ŠKuljević, P. \& Gomboc, S., 2014: Contribution to the knowledge of the butterfly fauna (Insecta: Lepidoptera) of the Adriatic island Hvar, Croatia. Acta Entomol. Slov. 22(1), 29-44.

Koren, T., Vukotić, K. \& Verovnik, R., 2015a: Butterflies (Lepidoptera: Papilionoidea \& Hesperioidea) of the Croatian islands: new surveys of Vir, Murter, Čiovo and Šolta. Entomol. Gaz. 66, 187-197.

Koren, T., Črne, M., Pavliha, G. \& Trkov, D., 2015b: Mountain Poštak, a new hotspot for the Lepidoptera of Croatia (Lepidoptera: Rhopalocera). SHILAP Revta. Lepidopterol. 43(169), 145-155.

Kosmač, M. \& Verovnik, R. 2009: First record of Cacyreus marshalli (Lycaenidae) from the Balkan Peninsula. Nota Lepidopterol. 32(1), 81-82.

Kučinić, M., Mihoci, I., Tvrtković, N., Šašıć, M., Bojanić, D., Joković, S., Mazija, M. \& Popijač, A., 2010: Butterfly Diversity (Insecta: Lepidoptera, Rhopalocera) of the Island of Mljet, Croatia. Proc..Symp. Branimir Gušić Days, Mljet, pp. 111-125.

Kučinić, M., Koren, T., Minoci, I., Vuković, M., Jakovljević, T. \& Jenčič, S., 2013: Can spreading of the Geranium Bronze Cacyreus marshalli (Butler, 1898) (insecta, Lepidoptera, Lycaenidae) in Croatia be assigned to climate change? Period. Biol. 115(3), 429-433.

Lafranchis, T., 2004: Butterflies of Europe. Diatheo, Paris.

Lorкоvić, Z., 1971: Gegenes nostrodamus F. and G. pumilio Hffgg. on the eastern Adriatic coast. Acta Entomol. Jug. 7(2), 56.

LoRкоvić, Z., 2009: The Rhopalocera fauna of Croatia with special respect to the fauna of Plitvice Lakes. Entomol. Croat. 13, 15-78.

MaGAš, D., 2013: Geografija Hrvatske. Manualia Universitatis Studiorum Jadertinae, Zadar.

Mıносі, I. \& ŠAšıć, M., 2006: Novi podaci o proširenju vrste Polyommatus thersites (Cantener, 1835) (Lepidoptera: Lycaenidae) u Hrvatskoj. Entomol. Croat. 10(2), 7-14.

Mıносі, I. \& Šššć, M., 2007: New distribution data on the endemic butterfly Proterebia afra dalmata (Godart, [1824]) (Nymphalidae, Satyrinae) in Croatia. Nat. Croat. 16(3), 205-210.

Mıносі, I., ŠAšıć, M. \& Vuкović, M., 2007: Contribution to the butterfly fauna (Hesperioidea \& Papilionoidea) of the Velebit Mountain, Croatia. Nat. Croat. 16(1), $29-62$.

Mihoci, I., Hršak, V., Kučinić, M., Mičetić Stanković, V., Delić, A. \& Tvrtković, N., 2011: Butterfly diversity and biogeography on the Croatian karst mountain Biokovo: Vertical distribution and preference for altitude and aspect? Eur. J. Entomol. 108, 623-633.

MANN, J., 1869: Lepidopteren gesammelt während dreier reisen nach Dalmatien in den Jahren 1850, 1862 und 1868. Ver. Zool.-bot. Ges. Wien 19, 371-388.

Mladinov, L., 1965: Rezultati istraživanja faune Rhopalocera i Heterocera otoka Paga. Biol. glasnik 18, $37-48$.

MLAdinov, L., 1973: Lepidoptera (Rhopalocera) zbirki Hrvatskog narodnog zoološkog muzeja u Zagrebu. Hrvatski narodni zoološki muzej Zagreb, 7.

Mladinov, L., 1975: Lepidoptera- Hesperiidae (Rhopalocera) u zbirki Hrvatskog narodnog zooloskog muzeja u Zagrebu. Hrvatski narodni zoološki muzej Zagreb, 8.

Schwingenschuss, L. \& WAgNER, F., 1925: Beitrag zur Macro-Lepidopteren-Fauna Süddalmatiens insbesondere der Umgebung Gravosa's. Zeit. Österr. Entomol. Ver. 10(6), 53-57, 66-71, 78-82, 116-119.

Stauder, H., 1911: Beiträge zur Kenntnis der Makrolepidopteren-fauna der adriatischen Küstengebiete. Bol. Soc. Adriat. Sci. Nat. Trieste 25(2), 93-120.

STAUDER, H., 1913: Weitere Beiträge zur Kenntnis der Makrolepidopterenfauna der adriatischen Küstengebiete. Bol. Soc. Adriat. Sci. Nat. 27(1), 105-166.

StAuder, H., 1921: Die Schmetterlingsfauna der illyro-adriatischen festland- und inselzone (faunula illyro-Adriatica). Zeit. Wiss. Insektenbiol. 16(1/2), 16-23; (3/4), 43-49; (5/6), 101-108; (7/8), 143-153; (9/10), 166-176; (11/12), 219-224.

STAUDER, H., 1922: Die Schmetterlingsfauna der illyro-adriatischen festland- und inselzone(faunula illyro-Adriatica). Zeit. Wiss. Insektenbiol. 17(1/2), 14-21; (3/4), 58-64, (5/6), 83-92; (7/8), 135-147; (9/12), $165-176$.

STAUDER, H., 1923: Die Schmetterlingsfauna der illyro-adriatischen festland- und inselzone (faunula illyro-Adriatica.). Zeit. Wiss. Insektenbiol. 18(1/2), 10-18; (3/4), 58-68; (5/7), 106-114; (8/9), 187-202; (10/11), 253-267; (12), 317-327.

ŠAšrć-KLJAJo, M. \& Minoci, I., 2009: Znanstvena analiza vrsta noćnih i danjih leptira s Dodatka II Direktive o zaštiti prirodnih staništa i divlje faune i flore za potrebe izrade prijedloga potencijalnih NATURA 2000 područja. Hrvatski prirodoslovni muzej, Zagreb. 
ŠAšıć, M. \& MıносI, I., 2011: Annotated checklist of Croatian butterflies with vernacular names. Nat. Croat. 20(2), 425-436.

ŠAšıć, M., MıHocı, I. \& KučınIĆ, M., 2013: Crveni popis danjih leptira Hrvatske. Državni zavod za zaštitu prirode, Ministarstvo zaštite okoliša i prirode, Zagreb.

Tolman, T. \& Lewington, R., 2008: Butterflies of Britain \& Europe. Harper Collins Publishers, London.

Tóth, J. P. \& VARGA, Z., 2011: Inter- and intraspecific variation in the genitalia of the 'Melitaea phoebe group' (Lepidoptera, Nymphalidae). Zool. Anz. 250(3), 258-268.

Tvrtković, N., M. Šašić, M. Vuković, I. Minoci \& BJelić, M., 2012: Review of the butterfly fauna (Hesperioidea \& Papilionoidea) of the Dinara mountain range. Nat. Croat. 21(2), 471-481.

VerovniK, R., 2011: Butterflies (Lepidoptera: Rhopalocera) of the Croatian islands: an update on published records and new surveys of Pašman and Ugljan. Entomol.Gaz. 62, 251-263.

Wiemers, M., 1982: Tagfalterbeobachtungen in Dalmatien/Jugoslawien. Atalanta 13 (3), 217-224.

Withrington, D. \& Verovnik, R., 2008: Butterflies (Rhopalocera) of the Croatian islands. Entomol. Gaz. $59,3-25$.

Withrington, D., 2008: Butterflies of the island of Hvar, Croatia. Bull. Amat. Entomol. Soc. 67(480), 193-196.

ZAKŠEK, V., 2005: On the presence of Proterebia afra (Lepidoptera: Nymphalidae: Satyrinae) on the island of Pag, Croatia. Phegea 33(3), 118-120.

\title{
SAŽETAK
}

\section{Prilog poznavanju faune leptira kopnenog dijela sjeverne Dalmacije}

\author{
R. Verovnik, T. Koren \& G. Glavan
}

Unatoč povijesnim i recentnim faunističkim istraživanjima, fauna dnevnih leptira nekih dijelova Hrvatske još je uvijek nedovoljno istražena. Kopneni dio sjeverne Dalmacije jedna je od takvih regija, unatoč tome što su okolna područja relativno dobro istražena. Kako bismo popunili tu prazninu, istraživali smo faunu dnevnih leptira na 25 lokacija raspoređenih od Vranskog jezera do Novigrada i Karinskog mora na istoku (sl. 1). Većina lokacija izabrana je prije terena uporabom Google Earth zračnih snimki.

Sveukupno smo tijekom istraživanja opazili 81 vrstu leptira, što je relativno niska raznolikost u usporedbi sa susjednim regijama Velebita i doline rijeke Zrmanje. Zabilježili smo nekoliko rijetkih i potencijalno ugroženih vrsta, čiju smo rasprostranjenost detaljnije raspravili. Moguće je da dvije vrste debeloglavaca, Carcharodus orientalis i Gegenes pumilio dosežu sjevernu granicu rasprostranjenosti u regiji, iako je potonja vrsta vjerojatno lutajuća bez stalnih kolonija na istraživanome području. Tri dodatne vrste, Euchloe ausonia, Tarucus balkanica i Hyponephele lupina također se nalaze blizu svoje sjeverne granice u Hrvatskoj, s tek nekoliko nalaza sjevernije. U radu prikazujemo i nove nalaze rijetkih vrsta poput Polyommatus thersites i Proterebia afra. Cacyreus marshalli, alohtona vrsta leptira u Europi je također opažena u sjevernoj Dalmaciji, ali nije česta.

Močvarna riđa (Euphydryas aurinia) nestala je s nekih istraživanih lokaliteta, no unatoč tome u sjevernoj Dalmaciji još uvijek postoje jake populacije. To je jedina vrsta s Direktive o staništima prisutna u regiji, i populacije u dobrom stanju mogu biti razlog za proglašavanje Natura 2000 lokacije.

Općenito, raznolikost faune leptira i vrijednost očuvanja regije nisu izrazito visoki, ali nekoliko lokacija sadrži većinu vrsta u regiji, uključujući i neke ugrožene vrste (sl. 2). Posebice doline rijeka Bijeline i Karišnice mogu biti određene kao mikrorezervati za zaštitu visoke raznolikosti leptira. Napuštanje i naknadno zaraštanje, zajedno s uništavanjem staništa radi izgradnje infrastrukture i širenja urbanih područja, prepoznati su kao najbitniji faktori koji uzrokuju opadanje raznolikosti leptira u regiji. 